\title{
A Neural Network Based Irradiance Meter
}

\author{
Akhil S M \\ Department of Electronics and Communication Engineering \\ MIT, Anjarakandy, Kannur, Kerala, India
}

\begin{abstract}
This paper proposes a novel design for a Low-Cost Sensor to measure Solar Irradiance. There are mainly three types of devices to measure solar irradiance over the Earth's surface: Pyranometers (direct and diffuse solar irradiance), Pyrheliometers (direct solar irradiance) and Albedometers (reflected solar irradiance). These devices measure irradiance in W/m2 being highly accurate and therefore expensive. The proposed sensor has to be able of measuring global and diffuse irradiance ensuring high precision. The device a good candidate for large deployment in photovoltaic power plants. The proposed sensor is implemented through a photovoltaic cell, a temperature sensor, and a low-cost microcontroller this paper proposes a novel approach for solar irradiance measurement based on neural networks. In the case of irradiance sensor which is available in market has high cost to calibrate, but in this case the use of a microcontroller allows for easy calibration, updates, and enhancement by simply adding code libraries.
\end{abstract}

Keywords: Neural networks, photovoltaic cells, pyranometer, sensor systems, solar irradiance

\section{Introduction}

PV systems are rapidly expanding and have increasing roles in electric power technologies, providing more secure power sources and pollution-free electric supplies. The operation planning of power systems including PV systems, the accurate prediction of the maximum power from the PV systems is inevitable. The maximum power generation depends on the environmental factors, mainly the irradiation and the cell temperature. As it is known, the output power in a solar cell is directly related to the climate conditions. Solar irradiance is a measure of how much solar power is getting at location. This irradiance varies throughout the year depending on the seasons. It also varies throughout the day depending on the position of the sun in sky, and weather. Solar irradiance is a key parameter for maximum power point (MPP) tracking (MPPT) in photovoltaic (PV) power plants and it relate to the solar cell itself. Each solar cell has a point at which current (I) and voltage (v) output from cell result in the maximum power output of the cell. Several mathematical models have been developed to estimate the behavior of the photovoltaic (PV) panels. The PV array's operating point is regulated by adjusting the duty cycle of the power converter connected at the output of the array. Temperature sensing is easy, however, measuring solar irradiance is difficult because irradiance sensors are expensive and difficult to calibrate. Previous methods are not flawless; they may oscillate around the MPP and can fail under rapidly changing irradiance conditions. In order to overcome these limitations, several papers have recently appeared in the literature based on neural networks (NN) and on approximate solutions. So here introducing a new method of calculating and increasing the efficiency of a solar system in low cost manner using neural network.

The rest of the paper is organized as follows: Section II illustrates the architecture of the proposed system. Section III describes Smart LED lighting system development methodology. Section IV showcases the software and hardware requirements of system development, and the paper discussion is concluded in Section V.

\section{Methodology}

Figure below shows the basic block diagram for the irradiance sensor using simple microcontroller. One or a small number of PV cells can be arranged in series/parallel in order to form a small sensing PV panel, which is connected to a known testing resistor, Rtest. The approach consists in measuring the voltage across the cell terminals and cell temperature.

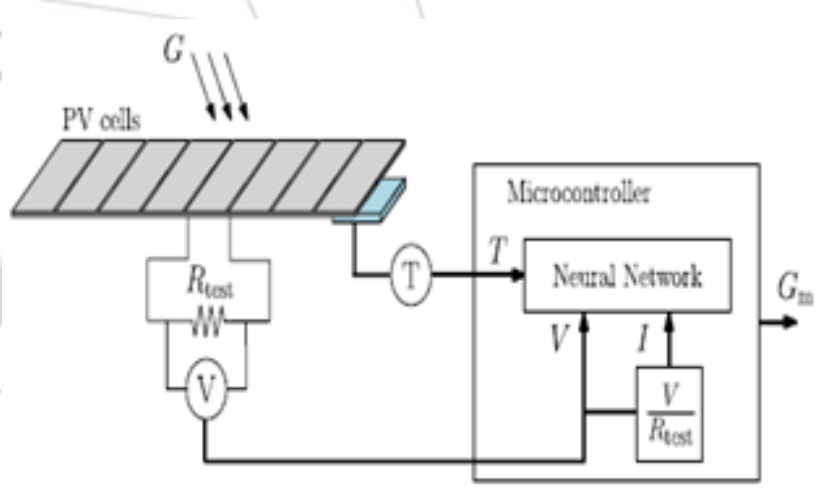

Figure (a): Basic approach of sensor

$\mathrm{NN}$ algorithm implemented in a low-cost microcontroller, which computes the actual solar irradiance with great accuracy. This sensor can be conveniently located next to a power producing PV panel or even integrated with it. This features the advantage that the sensing PV panel will "see" exactly the same solar irradiance and temperature as that "seen" by the power-producing PV panel, leading to improved performance.

By changing the in the parameters towards the neural network the accuracy of the overall system can be increased. Ie , also there considering voltage, current, temperature and as well as there is using humidity etc.

By adding the booster circuit will help to boost up the power when storing the power to a battery. The main disadvantage of this basic approach is the panel is fixed in one place. 


\section{International Journal of Science and Research (IJSR) \\ ISSN (Online): 2319-7064}

Index Copernicus Value (2013): 6.14 | Impact Factor (2015): 6.391

\section{A. Sun Movement:}

Sun is the center of solar system where the earth revolves around the sun in elliptical orbits. Time it takes the earth to encircle the sun is 1 year, or about 365 days. When the earth rotates does not exactly always on axis, but it has a slope varying from $0 \mathrm{o}$ to $23.45 \mathrm{o}$. The effect variation of the slope is changing seasons on the earth's surface. The earth orbit to the sun will form an angle position of the sun's equator (declination, $\delta$ ), which it can be calculated with Cooper equation as forth.

$$
\delta=23,45^{\circ} \sin \left[360^{\circ} x \frac{284+n}{365}\right]
$$

Where $\delta$ is the declination of sun, and $\mathrm{n}$ is the sequence number of days in a year. The resulting of earth rotation is apparent motion of the sun, which the sun as if appear in the sky to shift from east to west. Moreover, distributed temperature on the earth surface, the change of day and night as indicate time in the daily is 24 hours. In one earth's rotation is equal to $360 \mathrm{o}$ hence a shift of sun elevation angle is 150 per hour, while a shift of azimuth angle is 7.810 per month.

So there is adding a 2 axial solar tracking system and a sun tracking elements with this tracking panel can increase the overall efficiency. Manily these systems are used in the solar power plants also to prepare the irradiance map in the case of whether broadcasting systems. So there needed a wireless monitoring field to monitoring and controlling the whole system performance and also needed computer to store a measured data. There is also setting a lab view to control and store the observed values. Finally the overall block diagram of the system is shown in below

\section{B. Configuration of Artificial Neural Network}

The computation of solar irradiance through a NN has been chosen because of its versatility. Although several algorithms for multivariate fitting exist, they usually need data made on regular grids whereas NNs can perform multidimensional fitting by using any data distribution.

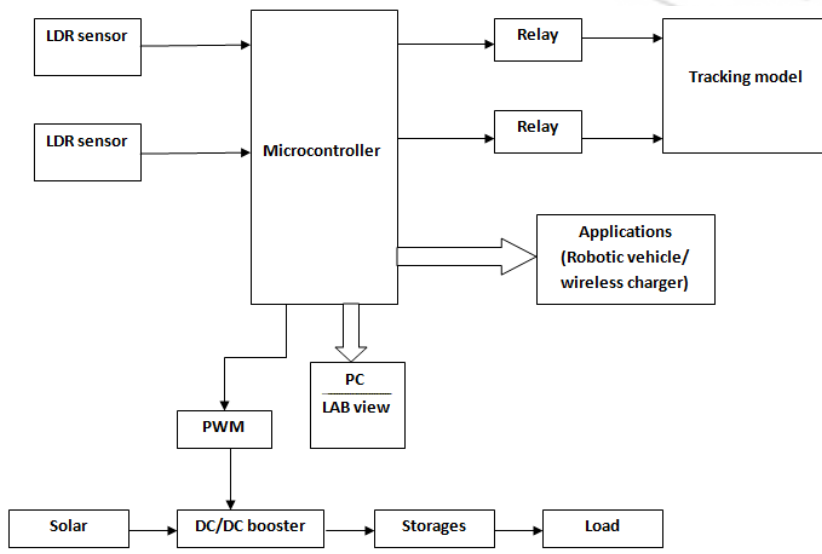

Figure (b): overall block diagram of the sensor

Moreover, the NN is a paradigm that can be realized by using simulation software, analog circuits, optical Microsystems and, in the case of this paper, microcontrollers. As discussed in this section, a feedforward NN (FFNN) with a single hidden layer has been chosen due to reasonable computational cost and its simple structure suitable to be embedded into an inexpensive eight-bit microcontroller. This is in contrast to other effective NN implementations, such as Bridged Multilayer Perceptron (BMLP) or fully connected cascade (FCC) architectures. Both BMLP and FCC require the implementation of a more elaborate structure and may bring actual benefits for problems involving a large number of neurons.

\section{Preparing the Training-Set}

The NN training process and hence the performance of the sensor proposed herein relies on a detailed knowledge of the PV array's I-V performance curves for different irradiance and temperature values. Performance $\mathrm{I}-\mathrm{V}$ curves for PV cells can be obtained using their mathematical model along with standard information provided by manufacturer's datasheets. Alternatively, operating points may be experimentally generated in the laboratory under controlled environmental conditions. In either case, a suitable training-set must be constructed by using $\mathrm{N}$ different $\mathrm{I}-\mathrm{V}$ curves depending on the irradiance using a constant temperature. Likewise, $\mathrm{M}$ different $\mathrm{I}-\mathrm{V}$ curves must be obtained by varying the temperature at a constant irradiance. Thus, $\mathrm{N} \times \mathrm{M}$ training set patterns must be created. This section provides a detailed description of I-V curves' generation from manufacturer's datasheets and an overview on the experimental synthesis.

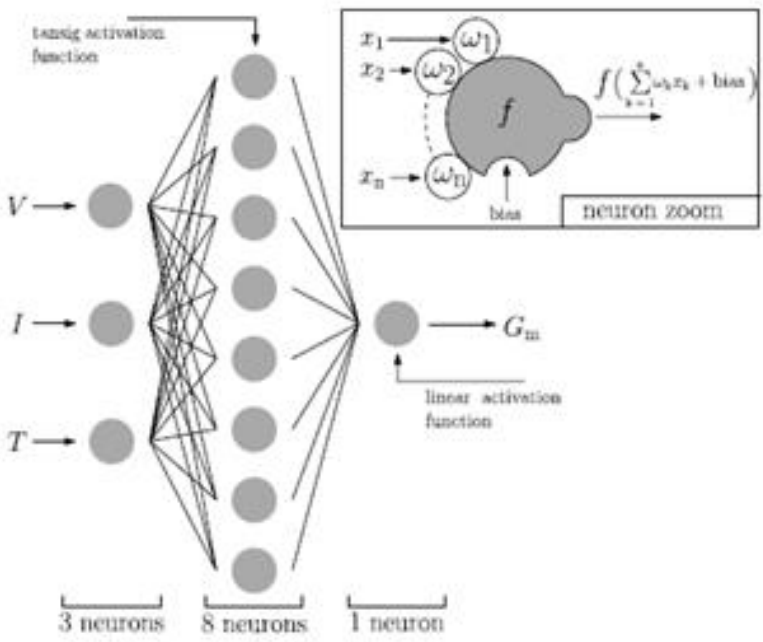

Figure (c): Architecture of proposed FNN scheme

The NN's training set was implemented in the MATLAB environment through $\mathrm{N}=10$ different operating points at equidistant irradiance values from 100 to $1500 \mathrm{~W} / \mathrm{m} 2$ and $\mathrm{M}=5$ different operating points at equidistant temperature values from 260 to $360(\mathrm{~K})$, which leads to a training set size of $\mathrm{N} \times \mathrm{M}=50$ operating points [V, I, T ]. The goal for mean square error (MSE) on training set was fixed equal to $8 \times 10^{-3}$ and the whole learning process took around 2min. Fig. (d) Shows the MSE during the NN training and validation. 


\section{International Journal of Science and Research (IJSR) \\ ISSN (Online): 2319-7064}

Index Copernicus Value (2013): 6.14 | Impact Factor (2015): 6.391

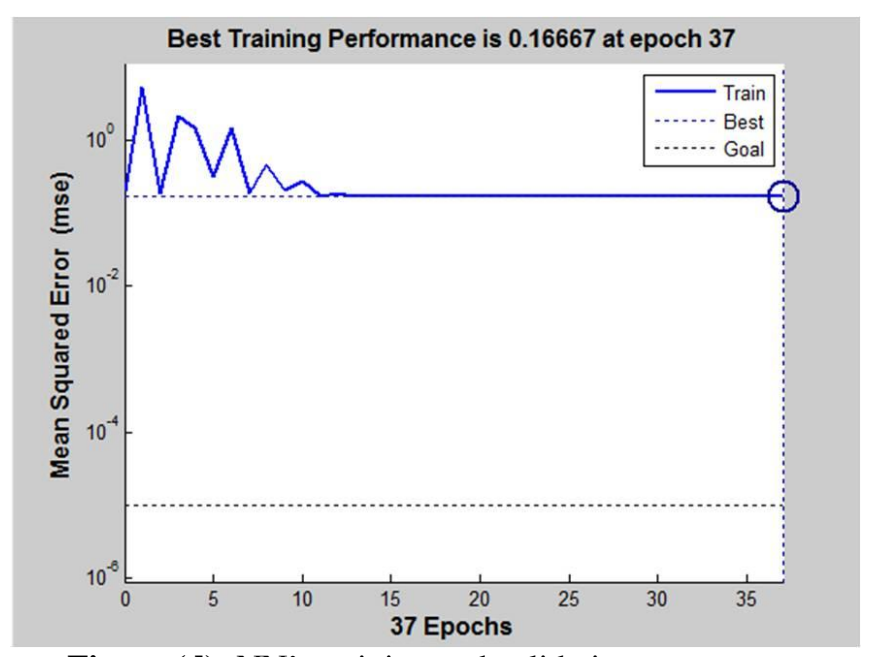

Figure (d): NN's training and validation processes

\section{Applications}

The solar panels are installed throughout the world widely. So the solar based applications are most commonly and very use full technology these days. If utilizing the solar energy correctly then we can get a good low cost energy as compared with electricity. Here there developed a sensor for monitoring the solar irradiance sensor using neural network by observing temperature, voltage, and current as training data's.

This solar irradiance sensor is mainly used in home appliances where solar panel is the major part. Also this sensor is mainly used in the weather broadcasting for the prediction of the power of irradiance. And also this sensor used for creation of the irradiance map of a particular geographical area. This sensor used in a heavy solar power plant irradiance sensor is used for the performance analysis. By connecting the overall system to internet then can send the weather and irradiance details to any mobile devices or remote devices.

\section{Conclusion}

This paper has introduced a novel low-cost NN-based solar irradiance sensor conceived to be utilized in large PV power plants for precise tracking of solar irradiance within the PV power plant layout. A complete mathematical model as well as a practical realization of the sensor has been described in detail, and outdoor measurements have been used to validate the sensor. An important feature of the approach proposed herein lies in the simplicity of construction along with a high degree of accuracy, thanks to the use of a NN implemented in a embedded system through a microcontroller. The NN was implemented through simple feed-forward architecture with a single hidden layer. The selection of this architecture was motivated by its reasonable computational cost and simple structure suitable to be embedded into an inexpensive eight-bit microcontroller. An interesting area of further research could be a comparison among different types of NNs as an evolution of this paper. The use of a microcontroller allows for updates, enhancement, and customization by simply adding code libraries. Moreover, it can be interfaced via RS232 or RS485 with other instruments or connected via Ethernet and remote controller through its embedded web server. A chief advantage of this sensor is that it can be conveniently located next to a power producing PV panel or even integrated with it. As a result, the sensing PV panel will "see" exactly the same solar irradiance and temperature as what is "seen" by the power-producing PV panel, leading to precise irradiance tracking thereby improving MPPT performance.

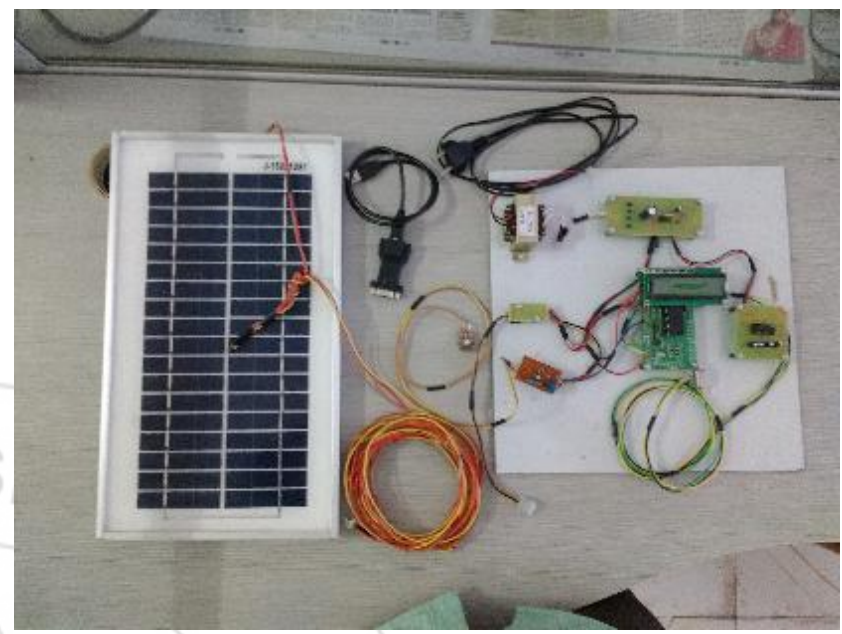

Figure (e): basic solar irradiance sensor implementation

\section{Acknowledgement}

First and foremost I thank the Almighty God for all the blessings. Special thanks to my college principal, dean and management for providing me with excellent library which were absolutely necessary for the completion of the work. I extend my heartfelt gratitude to Asst. Prof. Poornima Purushothaman for her whole hearted support, advice and suggestions as a guide to the work.

\section{References}

[1] "Neural Network Based Estimation of Maximum Power Generation from PV Module Using Environmental Information”. Takashi Hiyama, Senior Member IEEE, Ken KitabayashiDept. of Electrical Engineering \& Computer Science Kumamoto University, Kumamoto 860, Japan

[2] "Neural-Network-Based MPPT Control of a StandAlone Hybrid Power Generation System" Whei-Min Lin, Member, IEEE, Chih-Ming Hong, and ChiungHsing Chen

[3] "Design of a Low Cost Irradiance Meter using a Photovoltaic Panel" Joel Cruz-Colon, Luis MartinezMitjans, and Eduardo I. Ortiz-Rivera, Member, IEEE University of Puerto Rico-Mayagüez, ECE Department, eduardo.ortiz7@upr.edu

[4] "Microcontroller-Based Two-Axis Solar Tracking System" Lwin Lwin Oo Hardware Technology Department Computer University (Lashio) Myanmar 958-223969 1winlwinoo.72@gmail.com Nang Kaythi Hlaing Hardware Technology Department Computer University (Pinlone) Myanmar 958-130453 nangkthi.23@gmail.com

[5] "Development of Two Axis Solar Tracking Using 


\section{International Journal of Science and Research (IJSR) \\ ISSN (Online): 2319-7064}

Index Copernicus Value (2013): 6.14 | Impact Factor (2015): 6.391

Five Photodiodes" Munnik Haryanti Electrical Engineering Department Indonesia University Depok, Indonesia munnikbgr@yahoo.com Abdul Halim Electrical Engineering Department Indonesia University Depok, Indonesia halim_aha@yahoo.com Arbai Yusuf Satellite Technology Center Indonesian National Institute of Aeronautics and Space Bogor, Indonesia arbaibrwlpn@yahoo.com 